\title{
Co-evolution spreading of multiple information and epidemics on two-layered networks under the influence of mass media
}

\author{
Zhishuang Wang $\cdot$ Chengyi Xia
}

Received: 20 July 2020 / Accepted: 12 October 2020 / Published online: 2 November 2020

(C) Springer Nature B.V. 2020

\begin{abstract}
During epidemic outbreaks, there are various types of information about epidemic prevention disseminated simultaneously among the population. Meanwhile, the mass media also scrambles to report the information related to the epidemic. Inspired by these phenomena, we devise a model to discuss the dynamical characteristics of the co-evolution spreading of multiple information and epidemic under the influence of mass media. We construct the co-evolution model under the framework of two-layered networks and gain the dynamical equations and epidemic critical point with the help of the micro-Markov chain approach. The expression of epidemic critical point show that the positive and negative information have a direct impact on the epidemic critical point. Moreover, the mass media can indirectly affect the epidemic size and epidemic critical point through their interference with the dissemination of epidemic-relevant information. Though extensive numerical experiments, we examine the accuracy of the dynamical equations and expression of the epidemic critical point, showing that the dynamical characteristics of co-evolution spread-
\end{abstract}

\footnotetext{
Z. Wang · C. Xia $(\varangle)$

Tianjin Key Laboratory of Intelligence Computing and Novel Software Technology, Tianjin University of Technology, Tianjin 300384, China

e-mail: cyxia@email.tjut.edu.cn

Z. Wang · C. Xia

The Engineering Research Center of Learning-Based Intelligent System, Ministry of Education, Tianjin, China
}

ing can be well described by the dynamic equations and the epidemic critical point is able to be accurately calculated by the derived expression. The experimental results demonstrate that accelerating positive information dissemination and enhancing the propaganda intensity of mass media can efficaciously restrain the epidemic spreading. Interestingly, the way to accelerate the dissemination of negative information can also alleviate the epidemic to a certain extent when the positive information hardly spreads. Current results can provide some useful clues for epidemic prevention and control on the basis of epidemic-relevant information dissemination.

Keywords Epidemic spreading - Information dissemination - Two-layered networks · Mass media · MMC approach

\section{Introduction}

Varieties of information dissemination channels make it easier for people to obtain the information. In addition to the traditional way of disseminating information by word of mouth, we can also get information from online social networks, news websites and television stations, to name only a few. The dissemination of information has an increasingly profound impact on human society. In general, the epidemic spreading can trigger epidemic-relevant information to disseminate among the population [1]. People often make some 
responses to reduce the possibility of infection after gaining information about the epidemic, such as washing hand frequently and staying at home. As an example, for the COVID-19 (Coronavirus Disease 2019) [2-6], which is currently spreading globally, a considerable number of people chose to take some selfprotective actions against the epidemic after receiving the related information [7-9]. Therefore, the dissemination of epidemic-relevant information can have a certain degree of influence on the epidemic spreading [10-13]. It is of high importance to explore the characteristics of the co-evolution of epidemics and their related information in theory and practice [14].

The complex network theory proposed at the end of the twentieth century provides a brand new avenue to investigate the epidemic spreading and information dissemination [15-18], and a great quantity of researches on propagation dynamics have been gained under the framework of complex networks [19-23], but most of them have studied information dissemination and epidemic spreading separately [24-26]. For example, Pastor-Satorras and Vespignani discovered that critical threshold strikingly approaches zero when the network size of scale-free networks grows increasingly [27]; Liu et al. pointed out that networks with community structures are more conducive to epidemics outbreak than random networks [28]; Gross et al. indicated that the dynamic interaction of epidemic spread and network topology in adaptive networks can increase the epidemic threshold [29]. Moreover, Lü et al. concluded that small-world networks are more favorable for the information diffusion than random networks under the effect of social reinforcement [30]; Liu et al. presented that allowing the information to be diffused among the wider neighborhood can promote the dissemination of information [31].

In reality, human self-protective actions generated by epidemic-relevant information may greatly change the behavior of epidemics spreading, which has attracted extensive attention from researchers [32-38]. Funk et al. introduced the effect of epidemic-relevant awareness in the research of epidemics spreading, which suggests that the epidemic-relevant awareness is able to moderate the scale of the infectious disease [39]. Kiss et al. presented an improved SIRS (SusceptibleInfected-Recovered-Susceptible) model considering the related information dissemination, in which the effects of contact-based and population-based information diffusion as well as information attenuation on epidemic propagation are discussed [40]. It is worth noting that the network topologies of information dissemination and epidemic spreading are often different. For instance, in addition to word of mouth, information can also be disseminated through online social media, television, newspapers and so on. Hence, Granell et al. firstly constructed a two-layered multiplex networks to analyze the dynamics of the co-evolution of epidemic and its related information, which reveals that information dissemination can suppress the spread and outbreak of epidemics among the population [41].

Based on the framework of multi-layered networks, huge quantities of works have been carried out in recent years [42-48]. Considering the diversity of information communication channels, Granell et al. further discussed the inhibitory impact of information dissemination on the spread of SIS-type epidemics under the influence of mass media [49]. As a further step, Xia et al. discussed the role of mass media in the spread of SIR-like epidemics under the framework with twolayered networks [50]. It is worth noting that both of the aforementioned research efforts believed that only one type of epidemic-relevant information is spread among the population, which means that all individuals have the identical view on the prevention of epidemics. In practice, however, there is often more than one point of view on epidemic prevention among the population, and people may adopt different effects of self-protective actions based on their views when they realize the existence of epidemic. Take the COVID-19 as an example, a considerable number of individuals consider that this epidemic is quite serious and need to wear masks and maintain a social distance, but some still think that these self-protective actions are unnecessary. Meanwhile, people also often share their opinions with others based on their views on prevention of COVID-19 through social networks. Furthermore, during the outbreak of the epidemic, news related to the epidemic are also the focus of mass media coverage. Many people are informed about the epidemic for the first time through the mass media, but they could hold different views on these reports and take selfprotective actions with different effects. On one hand, these reports on the epidemic can prompt individuals to adopt more effective self-protective actions against the epidemic. On the other hand, related reports may render to people think that the media are too much nervous and it is not necessary to take such serious measures to 
cope with epidemics. The aforementioned differences in the individual perception of the severity of the epidemic and epidemic-relevant reports in the mass media may change the spreading behavior of the epidemic.

Thus, it is very significant to deeply explore how the dissemination of multiple epidemic-relevant information and the mass media reports affect the epidemic spreading. In this work, we investigate the dynamical characteristics of the co-evolution spreading of epidemics and two types of epidemic-relevant information under the influence of mass media under the framework of multiplex network. The main contribution of the current work can be summarized as follows.

(1) Based on the two-layered networks framework, we construct a co-evolution model for the co-evolution spreading of epidemics and two related information under the influence of mass media.

(2) We express the proposed co-evolution model into a mathematical form through micro-Markov chain (MMC) approach and analytically obtained the dynamical equations and the expression of the epidemic critical point.

(3) We examine the accuracy of the dynamical equations as well as the epidemic critical point expression, and discuss some dynamical characteristics of the proposed co-evolution model through a great quantity of simulation experiments.

The rest of this paper is structured as follows. Firstly, the proposed co-evolution model is constructed by using a framework of two-layered networks in Sect. 2. Then, the dynamical equations and the epidemic critical point expressions is analytically obtained with the help of MMC approach in Sect. 3. Next, though a great quantity of simulation experiments, the accuracy of the theoretical results is examined and some dynamical characteristics of the proposed co-evolution model are discussed in Sect. 4. Finally, some concluding remarks are provided in Sect. 5 .

\section{Model description}

In the proposed co-evolution model, we consider the impact of both two types of epidemic-relevant information and the related reports in the mass media on the epidemics spreading. It should be noted that these two types of epidemic-relevant information contain two different views about epidemic prevention. One

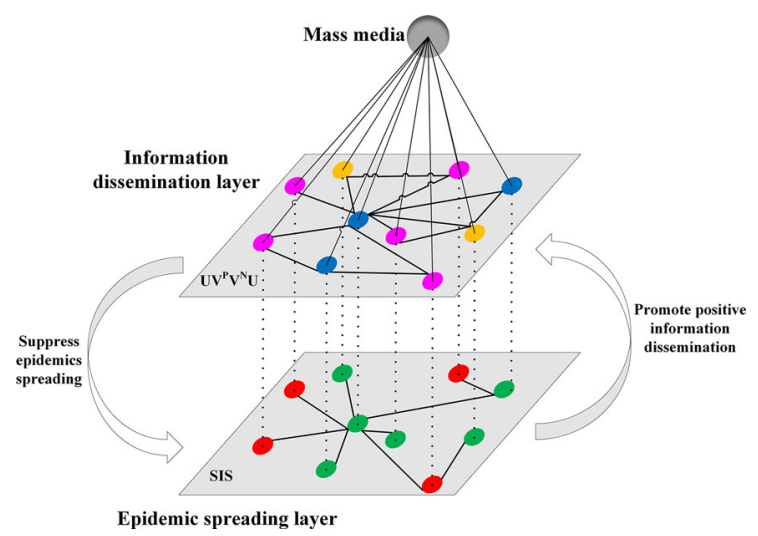

Fig. 1 Sketch of the proposed co-evolution model. The information dissemination layer indicates the potential information dissemination paths, while the epidemics can spread through these edges in the epidemic propagation layer. In the information dissemination layer, there are three states of $U$ (blue dots), $V^{P}$ (purple dots) and $V^{N}$ (yellow dots). In the epidemic spreading layer, there are two states of $S$ (green dots) and $I$ (red dots). (Color figure online)

view is that individuals need to take highly effective self-protective actions against the epidemic, and we term the information containing this view as positive information. Another view is that it is sufficient for individuals to take self-protective actions with poor effect against the epidemic, and we name the information containing this view as negative information. Meanwhile, the epidemic spreading can prompt individuals to take highly effective self-protective actions against the epidemic and disseminate positive information. With the help of the framework of two-layered networks, we constructed the co-evolution model as shown in Fig. 1, which contains three parts: mass media, information dissemination layer and epidemic spreading layer. Before explaining each part of the proposed coevolution model in detail, we enumerate the definitions of some symbols used in the proposed co-evolution model in Table 1.

As Fig. 1 shows, in the information dissemination layer, the network topology indicates the potential information dissemination paths related with epidemics (e.g., word of mouth or online social networks). We make use of an improved SIS epidemic model to characterize the epidemic-relevant information dissemination, which is called $U V^{P} V^{N} U$ model. There are three states $U, V^{P}$ and $V^{N}$ in the information dissemination layer. The state $U$ means that individuals do not know the epidemic-relevant information, while indi- 
Table 1 Definitions of some symbols used in the proposed co-evolution model

\begin{tabular}{|c|c|}
\hline Symbol & Definition \\
\hline$N$ & Total number of individuals in the proposed co-evolution model \\
\hline$A$ & Adjacency matrix of the network in the information dissemination layer \\
\hline$B$ & Adjacency matrix of the network in the epidemic spreading layer \\
\hline$a_{i j}$ & Elements of the adjacency matrix $A$ \\
\hline$b_{i j}$ & Elements of the adjacency matrix $B$ \\
\hline$m$ & Probability of an individual with state $U S$ receiving epidemic-relevant information through mass media \\
\hline$\eta$ & Probability of an individual with state $U S$ taking highly effective self-protective actions due to mass media \\
\hline$\kappa$ & Probability of an individual with state $V^{N} S$ changing his view on epidemic prevention due to mass media \\
\hline$\lambda_{P}$ & $\begin{array}{l}\text { Probability of an individual with state } U S \text { receiving positive information from one of his neighbors with state } V^{P} S \\
\text { or } V^{P} I\end{array}$ \\
\hline$\lambda_{N}$ & Probability of an individual with state $U S$ receiving negative information from one of his neighbors with state $V^{N} S$ \\
\hline$\xi$ & $\begin{array}{l}\text { Probability of an individual with state } V^{N} S \text { changing his view on epidemic prevention due to one of his neighbors } \\
\text { with state } V^{P} S \text { or } V^{P} I\end{array}$ \\
\hline$\delta_{P}$ & Forgetting rate of positive information \\
\hline$\delta_{N}$ & Forgetting rate of negative information \\
\hline$\beta$ & Epidemic infection rate \\
\hline$\Gamma_{P}$ & Attenuation factor of $\beta$ in case of taking highly effective self-protective actions \\
\hline$\Gamma_{N}$ & Attenuation factor of $\beta$ in case of taking self-protective actions with poor effect \\
\hline$\beta_{U}$ & Probability of an individual with sate $U S$ being infected by one of his neighbors with state $V^{P} I, \beta_{U}=\beta$ \\
\hline$\beta_{P}$ & Probability of an individual with sate $V^{P} S$ being infected by one of his neighbors with state $V^{P} I, \beta_{P}=\Gamma_{P} \beta$ \\
\hline$\beta_{N}$ & Probability of an individual with sate $V^{N} S$ being infected by one of his neighbors with state $V^{P} I, \beta_{N}=\Gamma_{N} \beta$ \\
\hline$\mu$ & Recovery rate of infected individuals \\
\hline$\omega$ & Preference of individuals with state $U S$ for negative information, $0 \leq \omega \leq 1$ \\
\hline
\end{tabular}

viduals in states $V^{P}$ and $V^{N}$ represent the disseminators of positive and negative information, respectively. These two types of information are often in a competitive relationship in the process of dissemination, that is, an individual can only recognize one of them at a time. We assume that when an individual receives two types of information at the same time, he will give priority to self-protective actions with poor effect to a certain extent due to the higher cost of the highly effective self-protective actions (e.g., time or money). The possible transitions among three states in the information dissemination layer are illustrated in panel (a) of Fig. 2.

In addition, individuals can also gain epidemicrelevant information through relevant reports in the mass media. In general, epidemic-relevant reports include the severity of the epidemic and some recommended protective measures. After receiving these reports about epidemics from the mass media, an individual with state $U$ will take appropriate self-protective

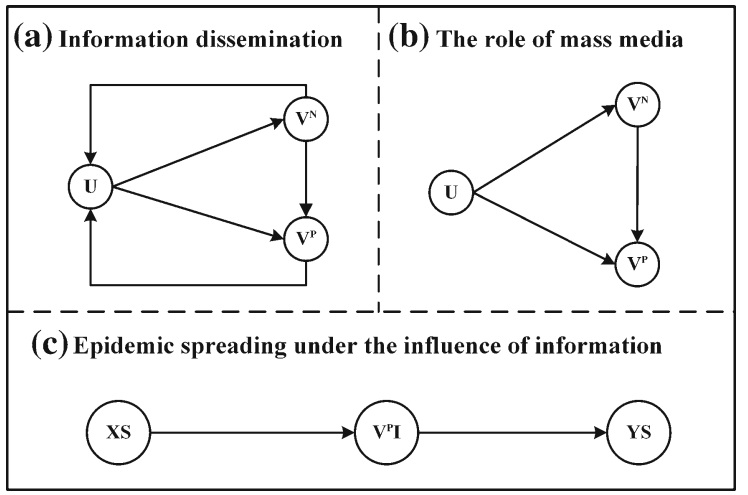

Fig. 2 Possible state transitions in the proposed co-evolution model. Panel a shows the possible state transitions in the information dissemination layer. Panel $\mathbf{b}$ shows the influence of mass media on the individual states in the information dissemination layer. Panel $\mathbf{c}$ shows the possible state transitions in the system when information dissemination and epidemic dissemination are considered together. In panel c, $X S, Y S \in\left\{U S, V^{P} S, V^{N} S\right\}$ 
actions against the epidemic based on their views on the severity of the epidemic, and further become a disseminator of positive or negative information. Moreover, enhancing the propaganda intensity of the mass media can encourage individuals with states $U$ to learn about the epidemics. The individuals with states $V^{N}$ may also change their views to take highly effective self-protective actions and become the disseminators of positive information due to the mass media's emphasis on highly effective prevention. The possible state transitions of individuals in information dissemination layer under the caused by mass media are illustrated in panel (b) of Fig. 2.

In the epidemic propagation layer, the edges between different nodes represent the mutual contact of individuals in the real world, and epidemics can spread through these edges. Each pair of nodes connected by dotted lines indicate that the same individual is in a different network. Meanwhile, we assume that all individuals are involved in epidemic spreading and information dissemination. We mainly concentrate on the impact of different kinds of epidemic-relevant information on SIS-type epidemics in the proposed coevolution model. Therefore, there are two states in the epidemic spreading layer: $S$ (Susceptible) and $I$ (Infected). Moreover, considering the coupling effect of epidemic spreading and corresponding information, the individuals in the proposed co-evolution model can be further divided into four states: $V^{P} S, V^{N} S, V^{P} I$ and $U S$. In addition, it is assumed that a susceptible is infected, he will immediately realize the necessity of taking highly effective self-protective actions and become a disseminator of positive information, that is, the epidemic spreading can prompt positive information dissemination by this means. Accordingly, we do not take the states $V^{N} I$ and $U I$ into account in the proposed co-evolution model since an infected individual will not forget the positive information before he is cured. The possible state transitions in the epidemic spreading layer under the influence of two types of information are illustrated in panel (c) of Fig. 2.

\section{Dynamical equations and epidemic critical point}

Referring to some previous researches, we make use of MMC approach to further analyze the dynamics of the co-evolution spreading with two types of epidemic-relevant information and epidemics in this section. First, the dynamical equations of the proposed co-evolution model are gained by means of MMC approach. Then, the epidemic critical point is analytically acquired by dealing with the dynamical equations.

\subsection{Dynamical equations}

According to the previous description in Sect. 2, the state of an individual $i$ may be one of $U S, V^{P} S, V^{N} S$ and $V^{P} I$ at each time step $t$, and the corresponding probabilities at these states are set to be $P_{i}^{U S}(t)$, $P_{i}^{V^{P} S}(t), P_{i}^{V^{N} S}(t)$ and $P_{i}^{V^{P} I}(t)$, respectively.

Considering that there may be more than one disseminator of positive or negative information among all neighbors of an individual, we use $1-r_{i}^{P}(t)$ and $1-r_{i}^{N}(t)$ to denote the probability that an individual with state $U S$ receives positive and negative information, respectively, where $r_{i}^{P}(t)$ and $r_{i}^{N}(t)$ can be represented as

$$
\left\{\begin{array}{l}
r_{i}^{P}(t)=\prod_{j}\left[1-a_{j i}\left(P_{j}^{V^{P} I}(t)+P_{j}^{V^{P} S}(t)\right) \lambda_{P}\right] \\
r_{i}^{N}(t)=\prod_{j}\left[1-a_{j i} P_{j}^{V^{N} S}(t) \lambda_{N}\right]
\end{array}\right.
$$

It is worth noting that an individual will only accept one view when he receives both positive and negative information. Therefore, we further introduce $\Theta_{i}^{P}(t)$ and $\Theta_{i}^{N}(t)$ to express the probability that an individual with state $U S$ agrees with the views in positive and negative information, respectively. Moreover, $\Theta_{i}^{U}(t)$ is used to represent that an individual with state $U S$ does not receive both types of information. Henceforth, $\Theta_{i}^{U}(t), \Theta_{i}^{P}(t)$ and $\Theta_{i}^{N}(t)$ can be defined as

$$
\left\{\begin{array}{l}
\Theta_{i}^{U}(t)=r_{i}^{P}(t) r_{i}^{N}(t) \\
\Theta_{i}^{P}(t)=1-r_{i}^{P}(t)-\omega\left(1-r_{i}^{P}(t)\right)\left(1-r_{i}^{N}(t)\right) \\
\Theta_{i}^{N}(t)=1-r_{i}^{N}(t)-(1-\omega)\left(1-r_{i}^{P}(t)\right)\left(1-r_{i}^{N}(t)\right)
\end{array}\right.
$$

An individual with status $V^{N} S$ can be affected by the disseminators of positive information in his neighbors to change his view of epidemic prevention and take highly effective self-protective actions, and the corresponding probability can be written as

$$
s_{i}^{P}(t)=\prod_{j}\left[1-a_{j i}\left(P_{j}^{V^{P} I}(t)+P_{j}^{V^{P} S}(t)\right) \xi\right] .
$$

Similarly, in the epidemic spreading layer, all infective neighbors of a susceptible individual may spread 


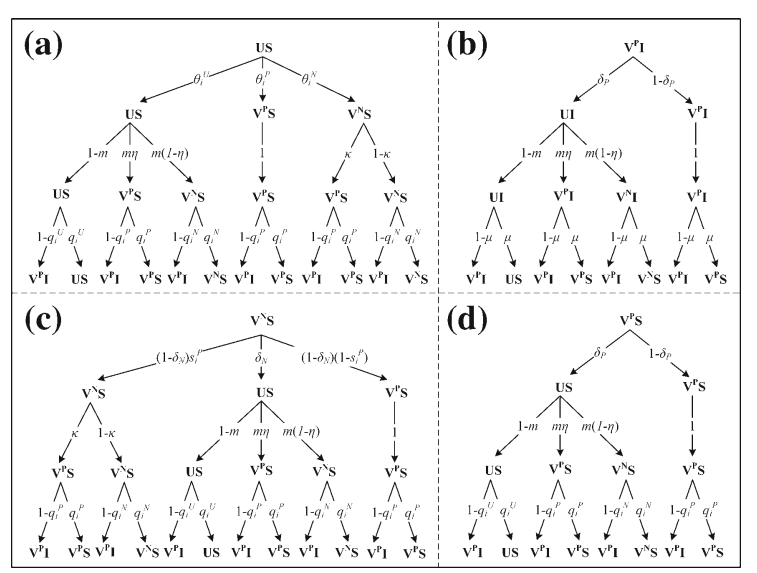

Fig. 3 State transition trees for the proposed co-evolution model. The root and leaf nodes of each tree represent the possible states of an individual at present and next time step, respectively. The parameters marked on the branches of each tree indicate the transition probabilities. In each panel, the first layer and the second layer represent the role of mass media and epidemic-relevant information dissemination in the states transition, and the third layer indicates the state transitions in the epidemic transmission layer under the influence of two types of epidemic-relevant information

the epidemic to him. Hence, the probabilities that individuals with states $V^{P} S, V^{N} S$ and $U S$ are not infected by all their neighbors with states $V^{P} I$ are represented as

$$
\left\{\begin{array}{l}
q_{i}^{P}(t)=\prod_{j}\left[1-b_{j i} P_{j}^{V^{P} I}(t) \beta_{P}\right] \\
q_{i}^{N}(t)=\prod_{j}\left[1-b_{j i} P_{j}^{V^{P} I}(t) \beta_{N}\right] \\
q_{i}^{U}(t)=\prod_{j}\left[1-b_{j i} P_{j}^{V^{P} I}(t) \beta_{U}\right]
\end{array} .\right.
$$

In order to depict the transitions between each state more clearly, we establish the state transition trees for the proposed co-evolution model in Fig. 3. In each panel of Fig. 3, the root node of a state transition tree characterizes the individual state at the present time step $t$, and the leaf nodes represent their possible states at the next time step. Afterwards, we can construct the dynamical equations for each individual in the proposed co-evolution model in light of the state transition trees as Eq. (5). Obviously, there are total $4 * N$ equations in Eq. (5). All these equations are used to calculate the probability that individual $i$ may be in each state at the next time step $\left(P_{i}^{V^{P} I}(t+1), P_{i}^{V^{P} S}(t+1)\right.$, $\left.P_{i}^{V^{N} S}(t+1), P_{i}^{U S}(t+1)\right)$, which are based on the current state $\left(P_{i}^{V^{P} I}(t), P_{i}^{V^{P} S}(t), P_{i}^{V^{N} S}(t), P_{i}^{U S}(t)\right)$ and the corresponding probability.

$$
\begin{aligned}
& \int P_{i}^{V^{P} I}(t+1)=P_{i}^{U S}(t)\left\{\Theta _ { i } ^ { U } ( t ) \left[(1-m)\left(1-q_{i}^{U}(t)\right)\right.\right. \\
& +m \eta\left(1-q_{i}^{P}(t)\right) \\
& \left.+m(1-\eta)\left(1-q_{i}^{N}(t)\right)\right] \\
& +\Theta_{i}^{P}(t)\left[1-q_{i}^{P}(t)\right] \\
& +\Theta_{i}^{N}(t)\left[\kappa\left(1-q_{i}^{P}(t)\right)\right. \\
& \left.\left.+(1-\kappa)\left(1-q_{i}^{N}(t)\right)\right]\right\} \\
& +P_{i}^{V^{P} S}(t)\left\{\delta _ { P } \left[(1-m)\left(1-q_{i}^{U}(t)\right)\right.\right. \\
& +m \eta\left(1-q_{i}^{P}(t)\right) \\
& \left.+m(1-\eta)\left(1-q_{i}^{N}(t)\right)\right] \\
& \left.+\left(1-\delta_{P}\right)\left[1-q_{i}^{P}(t)\right]\right\} \\
& +P_{i}^{V^{N} S}(t)\left\{\delta _ { N } \left[(1-m)\left(1-q_{i}^{U}(t)\right)\right.\right. \\
& +m \eta\left(1-q_{i}^{P}(t)\right) \\
& \left.+m(1-\eta)\left(1-q_{i}^{N}(t)\right)\right] \\
& +\left(1-\delta_{N}\right)\left[1-s_{i}^{P}(t)\right]\left[1-q_{i}^{P}(t)\right] \\
& +\left(1-\delta_{N}\right) s_{i}^{P}(t)\left[\kappa\left(1-q_{i}^{P}(t)\right)\right. \\
& \left.\left.+(1-\kappa)\left(1-q_{i}^{N}(t)\right)\right]\right\} \\
& +P_{i}^{V^{P} I}(t)\left\{\delta_{P}[(1-m)(1-\mu)\right. \\
& +m \eta(1-\mu)+m(1-\eta)(1-\mu)] \\
& \left.+\left(1-\delta_{P}\right)(1-\mu)\right\} \\
& P_{i}^{V^{P} S}(t+1)=P_{i}^{U S}(t)\left[\Theta_{i}^{U}(t) m \eta q_{i}^{P}(t)\right. \\
& \left.+\Theta_{i}^{P}(t) q_{i}^{P}(t)+\Theta_{i}^{N}(t) \kappa q_{i}^{P}(t)\right] \\
& +P_{i}^{V^{P} S}(t)\left[\delta_{P} m \eta q_{i}^{P}(t)\right. \\
& \left.+\left(1-\delta_{P}\right) q_{i}^{P}(t)\right] \\
& +P_{i}^{V^{N} S}(t)\left[\delta_{N} m \eta q_{i}^{P}(t)+\left(1-\delta_{N}\right)\right. \\
& {\left[1-s_{i}^{P}(t)\right] q_{i}^{P}(t)} \\
& \left.+\left(1-\delta_{N}\right) s_{i}^{P}(t) \kappa q_{i}^{P}(t)\right] \\
& +P_{i}^{V^{P} I}(t)\left[\delta_{P} m \eta \mu+\left(1-\delta_{P}\right) \mu\right] \\
& P_{i}^{V^{N} S}(t+1)=P_{i}^{U S}(t)\left[\Theta_{i}^{U}(t) m(1-\eta) q_{i}^{N}(t)\right. \\
& \left.+\Theta_{i}^{N}(t)(1-\kappa) q_{i}^{N}(t)\right] \\
& +P_{i}^{V^{N} S}(t)\left[\delta_{N} m(1-\eta) q_{i}^{N}(t)\right. \\
& \left.+\left(1-\delta_{N}\right) s_{i}^{P}(t)(1-\kappa) q_{i}^{N}(t)\right] \\
& +P_{i}^{V^{P}} S(t)\left[\delta_{P} m(1-\eta) q_{i}^{N}(t)\right] \\
& +P_{i}^{V^{P} I}(t)\left[\delta_{P} m(1-\eta) \mu\right] \\
& P_{i}^{U S}(t+1)=P_{i}^{U S}(t) \Theta_{i}^{U}(t)(1-m) q_{i}^{U}(t) \\
& +P_{i}^{V^{P} S}(t) \delta_{P}(1-m) q_{i}^{U}(t) \\
& +P_{i}^{V^{N} S}(t) \delta_{N}(1-m) q_{i}^{U}(t) \\
& +P_{i}^{V^{P} I}(t) \delta_{P}(1-m) \mu
\end{aligned}
$$




$$
\begin{aligned}
& P_{i}^{U S}=P_{i}^{U S} \Theta_{i}^{U}(1-m)\left(1-\tau_{i}^{U}\right) \\
& +P_{i}^{V^{P}} S_{\delta_{P}}(1-m)\left(1-\tau_{i}^{U}\right) \\
& +P_{i}^{V^{N}} S_{\delta_{N}}(1-m)\left(1-\tau_{i}^{U}\right) \\
& +\epsilon_{i} \delta_{P}(1-m) \mu \\
& P_{i}^{V^{P} S}=P_{i}^{U S}\left[\Theta_{i}^{U} m \eta\left(1-\tau_{i}^{P}\right)\right. \\
& \left.+\Theta_{i}^{P}\left(1-\tau_{i}^{P}\right)+\Theta_{i}^{N} \kappa\left(1-\tau_{i}^{P}\right)\right] \\
& +P_{i}^{V^{N} S}\left[\delta_{N} m \eta\left(1-\tau_{i}^{P}\right)\right. \\
& +\left(1-\delta_{N}\right)\left(1-s_{i}^{P}\right)\left(1-\tau_{i}^{P}\right) \\
& \left.+\left(1-\delta_{N}\right) s_{i}^{P} \kappa\left(1-\tau_{i}^{P}\right)\right] \\
& +P_{i}^{V^{P} S}\left[\delta_{P} m \eta\left(1-\tau_{i}^{P}\right)\right. \\
& \left.+\left(1-\delta_{P}\right)\left(1-\tau_{i}^{P}\right)\right] \\
& +\epsilon_{i}\left[\delta_{P} m \eta \mu+\left(1-\delta_{P}\right) \mu\right] \\
& P_{i}^{V^{N} S}=P_{i}^{U S}\left[\Theta_{i}^{U} m(1-\eta)\left(1-\tau_{i}^{N}\right)\right. \\
& \left.+\Theta_{i}^{N}(1-\kappa)\left(1-\tau_{i}^{N}\right)\right] \\
& +P_{i}^{V^{N} S}\left[\delta_{N} m(1-\eta)\left(1-\tau_{i}^{N}\right)\right. \\
& \left.+\left(1-\delta_{N}\right) s_{i}^{P}(1-\kappa)\left(1-\tau_{i}^{N}\right)\right] \\
& +P_{i}^{V^{P} S}\left[\delta_{P} m(1-\eta)\left(1-\tau_{i}^{N}\right)\right] \\
& +\epsilon_{i}\left[\delta_{P} m(1-\eta) \mu\right] \\
& \epsilon_{i}=P_{i}^{U S}\left\{\Theta _ { i } ^ { U } \left[(1-m) \tau_{i}^{U}\right.\right. \\
& \left.+m \eta \tau_{i}^{P}+m(1-\eta) \tau_{i}^{N}\right]+\Theta_{i}^{P} \tau_{i}^{P} \\
& \left.+\Theta_{i}^{P}\left[\kappa \tau_{i}^{P}+(1-\kappa) \tau_{i}^{N}\right]\right\} \\
& +P_{i}^{V^{P} S}\left\{\delta _ { P } \left[(1-m) \tau_{i}^{U}\right.\right. \\
& \left.+m \eta \tau_{i}^{P}+m(1-\eta) \tau_{i}^{N}\right] \\
& \left.+\left(1-\delta_{P}\right) \tau_{i}^{P}\right\} \\
& +P_{i}^{V^{N} S}\left\{\delta _ { N } \left[(1-m) \tau_{i}^{U}\right.\right. \\
& \left.\left.+m \eta \tau_{i}^{P}+m(1-\eta) \tau_{i}^{N}\right)\right] \\
& +\left(1-\delta_{N}\right)\left(1-s_{i}^{P}\right) \tau_{i}^{P} \\
& \left.+\left(1-\delta_{N}\right) s_{i}^{P}\left[\kappa \tau_{i}^{P}+(1-\kappa) \tau_{i}^{N}\right]\right\} \\
& +\epsilon_{i}\left\{\delta_{P}[(1-m)(1-\mu)\right. \\
& +m \eta(1-\mu)+m(1-\eta)(1-\mu)] \\
& \left.+\left(1-\delta_{P}\right)(1-\mu)\right\}
\end{aligned}
$$

3.2 Critical point of the epidemic model

The probabilities $P_{i}^{V^{P} I}(t), P_{i}^{V^{P}} S(t), P_{i}^{V^{N} S}(t), P_{i}^{U S}(t)$ for each individual $i$ are updated with time step $t$ in accordance with Eq. (5). When $t$ is large enough, we can obtain stable solutions to $4 * N$ equations in Eq. (5), and they can be denoted as $P_{i}^{V^{P} I}, P_{i}^{V^{P} S}$, $P_{i}^{V^{N} S}$ and $P_{i}^{U S}(i \in\{1,2, \ldots, N\})$. Similarly, $\Theta_{i}^{U}(t)$, $\Theta_{i}^{P}(t), \Theta_{i}^{N}(t), s_{i}^{P}(t), q_{i}^{P}(t), q_{i}^{N}(t)$ and $q_{i}^{U}(t)$ are represented as $\Theta_{i}^{U}, \Theta_{i}^{P}, \Theta_{i}^{N}, s_{i}^{P}, q_{i}^{P}, q_{i}^{N}$ and $q_{i}^{U}$ at steady state, respectively. The stable solutions of Eq. 5 can be employed to further derive the epidemic critical point for the proposed co-evolution model. Since the number of infective individuals in steady state is extremely few when $\beta$ is near the epidemic critical point, it can be supposed that $P_{i}^{V^{P} I}=\epsilon_{i} \ll 1$. Correspondingly, $q_{i}^{P}$, $q_{i}^{N}$ and $q_{i}^{U}$ can be approximated as follows,

$$
\left\{\begin{array}{l}
q_{i}^{P} \approx 1-\beta_{P} \sum_{j} b_{j i} \epsilon_{j}=1-\tau_{i}^{P} \\
q_{i}^{N} \approx 1-\beta_{N} \sum_{j} b_{j i} \epsilon_{j}=1-\tau_{i}^{N} \\
q_{i}^{U} \approx 1-\beta_{U} \sum_{j} b_{j i} \epsilon_{j}=1-\tau_{i}^{U}
\end{array},\right.
$$

where $\tau_{i}^{P}=\beta_{P} \sum_{j} b_{j i} \epsilon_{j}, \tau_{i}^{N}=\beta_{N} \sum_{j} b_{j i} \epsilon_{j}$ and $\tau_{i}^{U}=\beta_{U} \sum_{j} b_{j i} \epsilon_{j}$.

According to Eqs. (5) and (7), we can get the dynamical equations at steady state when $\beta$ is near epidemic critical point $\beta_{c}$, which can be expressed as Eq. (6). In the light of the definitions of $\epsilon_{i}, \tau_{i}^{P}, \tau_{i}^{N}$ and $\tau_{i}^{U}$, the first three terms in Eq. (6) can be approximated as follows,

$$
\begin{aligned}
& P_{i}^{U S}=P_{i}^{U S} \Theta_{i}^{U}(1-m)+P_{i}^{V^{P}} S_{\delta_{P}}(1-m) \\
& +P_{i}^{V^{N}} S_{\delta_{N}}(1-m) \\
& P_{i}^{V^{P} S}=P_{i}^{U S}\left(\Theta_{i}^{U} m \eta+\Theta_{i}^{P}+\Theta_{i}^{N} \kappa\right) \\
& +P_{i}^{V^{P}} S\left[\delta_{P} m \eta+\left(1-\delta_{P}\right)\right] \\
& +P_{i}^{V^{N} S}\left[\delta_{N} m \eta+\left(1-\delta_{N}\right)\left(1-s_{i}^{P}\right)\right. \\
& \left.+\left(1-\delta_{N}\right) s_{i}^{P} \kappa\right] \\
& P_{i}^{V^{N} S}=P_{i}^{U S}\left[\Theta_{i}^{U} m(1-\eta)+\Theta_{i}^{N}(1-\kappa)\right] \\
& +P_{i}^{V^{P} S_{\delta} m(1-\eta)} \\
& +P_{i}^{V^{N} S}\left[\delta_{N} m(1-\eta)+\left(1-\delta_{N}\right) s_{i}^{P}(1-\kappa)\right]
\end{aligned}
$$




$$
\begin{aligned}
\mu \epsilon_{i} & =P_{i}^{U S} \tau_{i}^{U}+P_{i}^{V^{P} S} \tau_{i}^{P}+P_{i}^{V^{N} S} \tau_{i}^{N} \\
& =\beta_{U}\left(P_{i}^{U S}+P_{i}^{V^{P} S} \Gamma_{P}+P_{i}^{V^{N} S} \Gamma_{N}\right) \sum_{j} b_{j i} \epsilon_{j}
\end{aligned}
$$

Let $P_{i}^{V^{P}}=P_{i}^{V^{P} S}+P_{i}^{V^{P} I} \approx P_{i}^{V^{P} S}$ and $P_{i}^{V^{N}}=$ $P_{i}^{V^{N} S}$, then Eq. (9) can be expressed as

$$
\begin{aligned}
\mu \epsilon_{i}= & \beta_{U}\left[1-\left(1-\Gamma_{P}\right) P_{i}^{V^{P}}\right. \\
& \left.-\left(1-\Gamma_{N}\right) P_{i}^{V^{N}}\right] \sum_{j} b_{j i} \epsilon_{j}
\end{aligned}
$$

There is no self-loop in the network of the epidemic spreading layer, that is, $b_{i j}=0$ when $i=j$. Additionally, let $\left[c_{i j}\right]$ be an identity matrix with $N * N$ elements. Hence, moving all the items on the right side of Eq. (10) to its left side, we can get

$$
\begin{aligned}
& \sum_{j} \epsilon_{j}\left\{\left[1-\left(1-\Gamma_{P}\right) P_{i}^{V^{P}}-\left(1-\Gamma_{N}\right) P_{i}^{V^{N}}\right] b_{j i}\right. \\
& \left.-\frac{\mu}{\beta^{U}} c_{j i}\right\}=0
\end{aligned}
$$

Then, the expression of the epidemic critical point can be represented as follows

$\beta_{c}=\frac{\mu}{\Lambda_{\max }(M)}$,

where $\Lambda_{\max }(M)$ is the greatest eigenvalue of the matrix $M=\left[m_{j i}\right]$ and $m_{j i}=\left[1-\left(1-\Gamma_{P}\right) P_{i}^{V^{P}}-(1-\right.$ $\left.\left.\Gamma_{N}\right) P_{i}^{V^{N}}\right] b_{j i}$.

Consequently, from the expression of the analyzed epidemic critical point, we can find that both positive and negative information have a direct impact on the epidemic critical point. Although the role of mass media is not reflected in the expression of the epidemic critical point, it can act indirectly on the epidemic critical point through the information dissemination layer. Furthermore, $P_{i}^{V^{P}}$ and $P_{i}^{V^{N}}$ in Eq. (12) can be calculated by Eq. (5) when the epidemic spreading does not interfere with the dissemination of the two types of epidemic-relevant information.

\section{Numerical simulations}

The simulation experiments for the proposed coevolution model are carried out under the framework of two-layered multiplex networks. In order to simulate the behavior of epidemic spreading, the BA (BarabásiAlbert) model is employed to generate the network characterizing the interaction between individuals. The size and average degree of scale free networks are 10000 and 6, respectively. Moreover, considering the diversification of information dissemination pathways (e.g., word of mouth and social media), we place additional 3000 edges into the generated BA network to represent the topology of the information dissemination network. The experimental results are gained by a multitude of Monte Carlo (MC) simulations and MMC calculations. Let $\rho^{I}, \rho^{V^{P}}$, and $\rho^{V^{N}}$ denote the densities of individuals with states $I, V^{P}$ and $V^{N}$ in the epidemic spreading layer and information dissemination layer, respectively. Furthermore, we suppose that the initial density for each realization is $\rho^{I}=\rho^{V^{P}}=\rho^{V^{N}}=$ 0.01 . To be specific, for each realization of MMC calculations, the initial value of each node in each state are $P_{i}^{U S}(t)=0.98, P_{i}^{V^{P} S}(t)=0, P_{i}^{V^{N} S}(t)=0.01$ and $P_{i}^{V^{P} I}(t)=0.01$ when $t=0$, respectively. In addition, at the beginning of each realization of MC simulations, we randomly select 200 nodes and initialize their states to $V^{P} I$ and $V^{N} S$, respectively. In other words, the initial number of nodes in states $U S, V^{P} S, V^{N} S$ and $V^{P} I$ are $9800,0,100$ and 100, respectively. It should be noted that all the experimental results acquired by MC simulations in this section are the average of the results of 50 realizations. In this section, the accuracy of dynamical equations obtained by MMC is examined at first; afterwards, the effects of epidemic-relevant information and mass media on epidemics spreading are discussed; and finally, the derived epidemic critical point is examined and analyzed.

\subsection{Comparison between MMC and MC}

We make use of MC simulations and MMC calculations to obtain the densities $\rho^{I}, \rho^{V^{P}}$ and $\rho^{V^{N}}$ under the same conditions, respectively, when the system tends to be steady. Afterwards, the accuracy of MMC calculations is evaluated by means of comparing two types of results, where the densities $\rho^{I}, \rho^{V^{P}}$ and $\rho^{V^{N}}$ acquired through two different methods are illustrated in Fig. 4. The comparison of each group of $\rho^{I}, \rho^{V^{P}}$ and $\rho^{V^{N}}$ shows that there are subtle differences between two methods. When $\beta$ exceeds the epidemic critical point, the dissemination of positive information can be further 

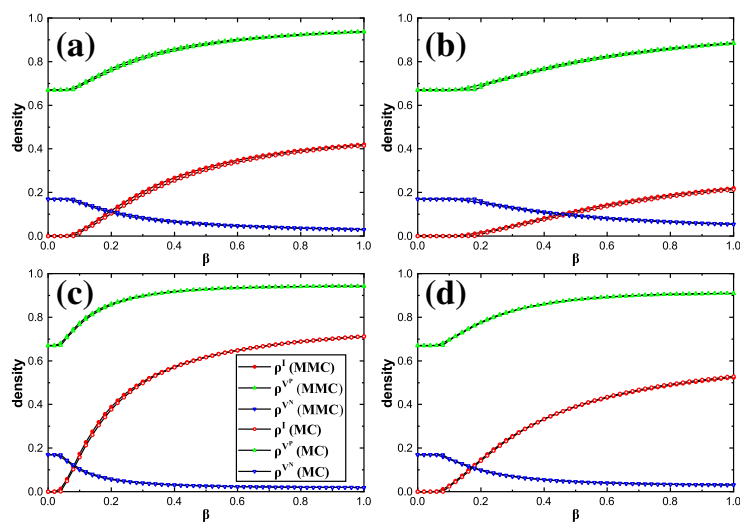

Fig. 4 Comparison of $\rho^{I}, \rho^{V^{P}}$ and $\rho^{V^{N}}$ gained by MMC and $\mathrm{MC}$ in the case of different infection rare $(\beta)$. The higher consistency between the solid and hollow lines of the same color indicates the higher accuracy of the dynamical equations. In panel $\mathbf{a}-\mathbf{d}$, the parameters $\left(\mu, \Gamma_{P}, \Gamma_{N}\right)$ are set to $(0.3,0,0.5)$, $(0.6,0,0.5),(0.3,0.3,0.8)$ and $(0.6,0.3,0.8)$, respectively. Other parameters in each panel are set as $\lambda_{P}=\lambda_{N}=0.5$, $\delta_{P}=\delta_{N}=0.3, m=0.4, \xi=0.2, \kappa=0.2, \eta=0.3$ and $\omega=1$. (Color figure online)

promoted by persistent infective individuals, while the dissemination of negative information that competes with the positive information is suppressed to a certain extent. Moreover, the differences of $\rho^{I}$ in each panel of Fig. 4 also demonstrate that the effectiveness of treatment and self-protection actions can play an important role in epidemic control. In addition, for the purpose of further validations, we also show the comparison of the density $\rho^{I}$ in the case of more parameters in Figs. 5 and 6. In order to more intuitively depict the similarity of the results gained by two methods, we calculate the mean value of the relative error of each group of comparison from Figs. 4, 5, 6, and they are illustrated in Tables 2 and 3. Accordingly, all these data in Tables 2 and 3 clearly prove that the dynamical equations based on MMC shown in Eq. (5) can well describe the dynamical characteristics of co-evolution spreading of epidemics and two types of related information under the influence of mass media in the proposed co-evolution model.

\subsection{The effect of information and mass media on epidemics spreading}

In addition to verifying the correctness of the results obtained by MMC, Figs. 5 and 6 also show the impact
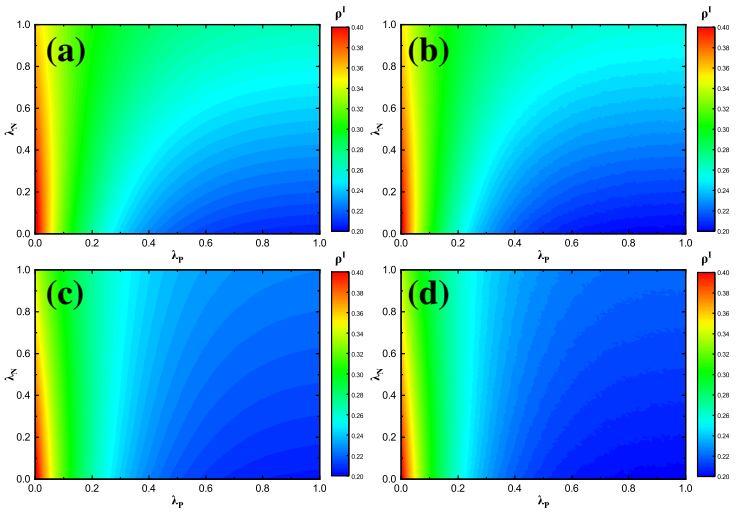

Fig. 5 Effect of $\lambda_{P}$ and $\lambda_{N}$ on density $\rho^{I}$. Each panel shows the epidemic size in the system under different combinations of $\lambda_{P}$ and $\lambda_{N}$. The results in panel $\mathbf{a}$ and panel $\mathbf{c}$ are calculated by MMC calculations. The results in panel $\mathbf{b}$ and panel $\mathbf{d}$ are gained by MC simulations. The consistency between the densities $\rho^{I}$ gained by the two means shows that the dynamical equations have higher accuracy. $\kappa$ is equal to 0.2 in panel $\mathbf{a}$ and $\mathbf{b}$, while it is 0.6 in panel $\mathbf{c}$ and $\mathbf{d}$. Other parameters in each panel are set to be $\beta=0.3, \mu=0.5, \delta_{P}=\delta_{N}=0.6, m=0.2, \xi=0.2$, $\eta=0.3, \omega=1, \Gamma_{P}=0$ and $\Gamma_{N}=0.5$

of two types of information dissemination and mass media on the epidemic size (i.e., the density $\rho^{I}$ ) at the steady state. We first focus on the influence of two information dissemination rates on epidemic size shown in Fig. 5. Taking panel (a) of Fig. 5 as an example, on the one hand, the density $\rho^{I}$ shows a downward trend as $\lambda_{P}$ increases little by little when $\lambda_{N}$ is fixed to any value in $[0,1]$. This is due to the fact that larger $\lambda_{P}$ can trigger the rapid dissemination of positive information, while the dissemination of negative information that competes with the positive information is suppressed to a certain extent. Accordingly, the proportion of individuals who can recognize the view of positive information will be higher at the steady state when $\lambda_{P}$ is a larger one, and more individuals taking highly effective self-protective actions can greatly reduce the epidemic size.

On the other hand, the influence of $\lambda_{N}$ on the density $\rho^{I}$ can be divided into two different situations according to the value of $\lambda_{P}$ in panel (a) of Fig. 5. When $\lambda_{P}$ is less than 0.07 , the density $\rho^{I}$ does not increase with the increase of $\lambda_{N}$ but shows a downward trend. There are two chief causes for this situation. One is that positive information is difficult to disseminate when $\lambda_{P}$ is small $\left(\lambda_{P}<0.07\right)$, and the infected individuals rarely distribute the positive information to their susceptible 


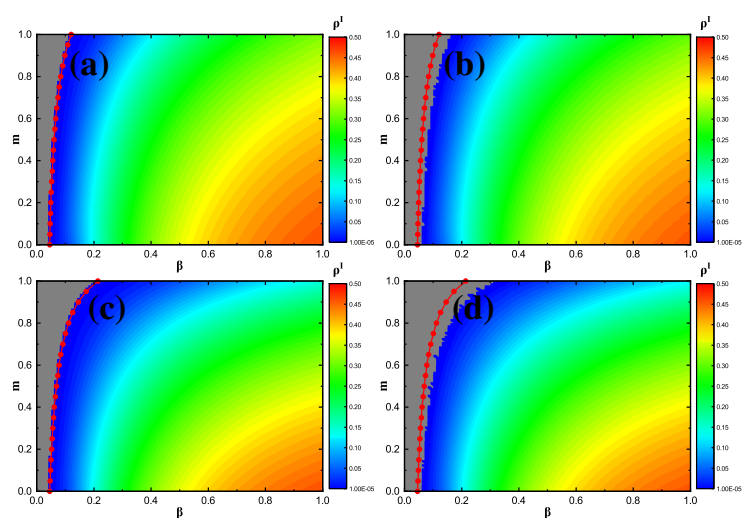

Fig. 6 Effect of $m$ and $\beta$ on density $\rho^{I}$. Each panel shows the epidemic size in the system under different combinations of $\beta$ and $m$. The results in panel $\mathbf{a}$ and panel $\mathbf{c}$ are calculated by MMC calculations. The results in panel $\mathbf{b}$ and panel $\mathbf{d}$ are gained by MC simulations. The consistency between the densities $\rho^{I}$ gained by the two means shows that the dynamical equations have higher accuracy. $\eta$ is equal to 0.3 in panel (a) and (b), while it is 0.6 in panel (c) and (d). Other parameters in each panel are $\mu=0.3, \lambda_{P}=\lambda_{N}=0.5, \delta_{P}=\delta_{N}=0.3$, $\kappa=0.2, \xi=0.2, \omega=1, \Gamma_{P}=0$ and $\Gamma_{N}=0.5$. The gray area in each panel indicates that $\rho^{I}=0$. The red line with the symbol represents the epidemic critical point calculated from Eq. (12). The changing trend of the red lines with the symbol demonstrate that the epidemic critical point can be accurately calculated from the derived expression. (Color figure online)

neighbors even if they agree that individuals need to take highly effective self-protective actions against the epidemic. That is, the epidemic spreading is scarcely influenced by positive information on condition that $\lambda_{P}$ is less than 0.07 . The other one is that more and more individuals with states US become conscious of taking self-protective actions with poor effect against the epidemic as $\lambda_{N}$ increases little by little. Although the self-protective actions with poor effect taken by these individuals are not effective enough, they can still suppress the epidemic spreading to a certain extent when positive information is difficult to disseminate. Nevertheless, when $\lambda_{P}$ is greater than 0.07 , the inversion phenomenon appears, in which density $\rho^{I}$ increases with $\lambda_{N}$. In particular, this phenomenon becomes more obvious when $\lambda_{P}$ is larger because the larger $\lambda_{N}$ creates a greater obstacle to the dissemination of positive information that competes with negative information. On the whole, increasing $\lambda_{P}$ and decreasing $\lambda_{N}$ are two considerable means to effectively control the epidemic.
Table 2 The mean value of the relative errors of each group of comparison in Fig. 4

\begin{tabular}{llll}
\hline & $\rho^{I}(\%)$ & $\rho^{V^{P}}(\%)$ & $\rho^{V^{N}}(\%)$ \\
\hline Figure 4a & 6.05 & 0.67 & 4.93 \\
Figure 4b & 6.51 & 0.71 & 3.52 \\
Figure 4c & 1.69 & 0.24 & 1.99 \\
Figure 4d & 1.92 & 0.19 & 1.35 \\
\hline
\end{tabular}

Table 3 The mean value of the relative errors of each group of comparison in Fig. 5 and Fig. 6

\begin{tabular}{lll}
\hline & $\begin{array}{l}\text { Panel (a) versus } \\
\text { panel (b) }(\%)\end{array}$ & $\begin{array}{l}\text { Panel (c) versus } \\
\text { panel (d) }(\%)\end{array}$ \\
\hline Figure 5 & 4.01 & 4.44 \\
Figure 6 & 6.78 & 8.11 \\
\hline
\end{tabular}

In panel (c) of Fig. 5, the change trend of density $\rho^{I}$ with $\lambda_{P}$ and $\lambda_{N}$ is similar to that in panel (a) of Fig. 5. The difference, however, is that density $\rho^{I}$ in panel (c) of Fig. 5 is smaller than that in panel (a) of Fig. 5 for any given set of $\lambda_{P}$ and $\lambda_{N}$. For instance, when $\lambda_{P}=0.5$ and $\lambda_{N}=0.6$, the corresponding values of density $\rho^{I}$ in panel (a) and panel (c) of Fig. 5 are 0.2555 and 0.2309 , respectively. This difference can be attributed to the impact of mass media on the spread of epidemics to some extent. The epidemic will be able to be further controlled when more and more disseminators of negative information realize the need for highly effective self-protective actions through the mass media.

Next, the effects of $\beta$ and $m$ on the density $\rho^{I}$ are discussed. In panel (a) of Fig. 6, the larger $m$ can reduce the density $\rho^{I}$ when $\beta$ exceeds the epidemic critical point. In particular, this situation is more apparent when the probability of epidemic spreading is relatively high. We can explain this situation from the perspective of the influence of mass media on the total number of individuals who are conscious of the existence of epidemic. When $\beta$ is fixed at a value greater than the epidemic critical point, the sum of $\rho^{V^{P}}$ and $\rho^{V^{N}}$ is positively correlated with $m$. Although the effectiveness of selfprotective actions with poor effect is lower than that of highly effective self-protective actions, the increase in the sum of $\rho^{V^{P}}$ and $\rho^{V^{N}}$ under the influence of 
mass media can still reduce the density $\rho^{I}$. Therefore, enhancing the propaganda intensity of the mass media is an effective means to alleviate the epidemic. Moreover, by analyzing the difference between panel (a) and panel (c) in Fig. 6, we can find that the density $\rho^{I}$ in panel (c) is relatively lower under the same conditions. As a consequence, more and more individuals realize the importance of highly effective self-protective actions under the influence of mass media, which is greatly conductive to the epidemic control.

\subsection{Discussion on epidemic critical point}

The epidemic critical point is one of the key quantities in epidemic propagation dynamics. In light of the expression in Eq. (12), we further discuss the epidemic critical point $\left(\beta_{c}\right)$ of the proposed co-evolution model. For the purpose of verifying the accuracy of the epidemic critical point expression, we compare the results calculated by Eq. (12) with those obtained by MMC calculations and MC simulations in Fig. 6. The red lines with symbol in each panel of Fig. 6 represent the epidemic critical points calculated by Eq. (12) in which $m$ takes different values. Moreover, the gray area in each panel of Fig. 6 means that $\rho^{I}=0$, and the other color areas indicate that $\rho^{I}>0$. Taking together, $\beta$ corresponding to the boundary between the gray area and other color areas can be regarded as $\beta_{c}$. According to panel (a) and (c) of Fig. 6, it can be observed that $\beta_{c}$ calculated by Eq. (12) is consistent with the $\beta_{c}$ obtained by the MMC calculations. In addition, although there is a slight difference between the results calculated by Eq. (12) and those obtained by MC simulations, the change trend of these two results is consistent.

Based on Fig.6a and c, it can be found that a larger $m$ will result in a larger $\beta_{c}$, particularly when $\eta=0.6$ (the results in panel (c)). Similarly, this situation is also shown in Fig. 7. In each panel of Fig. 7, $\beta_{c}$ is positively correlated with $m$ no matter what value $\mu$ takes, and the influence of $m$ on $\beta_{c}$ becomes more and more obvious as $\mu$ increases. The aforementioned variations in $\beta_{c}$ with $m$ demonstrate that increasing the propaganda intensity of the mass media and highlighting the importance of taking highly effective self-protective actions in publicity can play a positive role in the prevention of outbreaks. Meanwhile, these results also demonstrate that the mass media can indirectly influ-
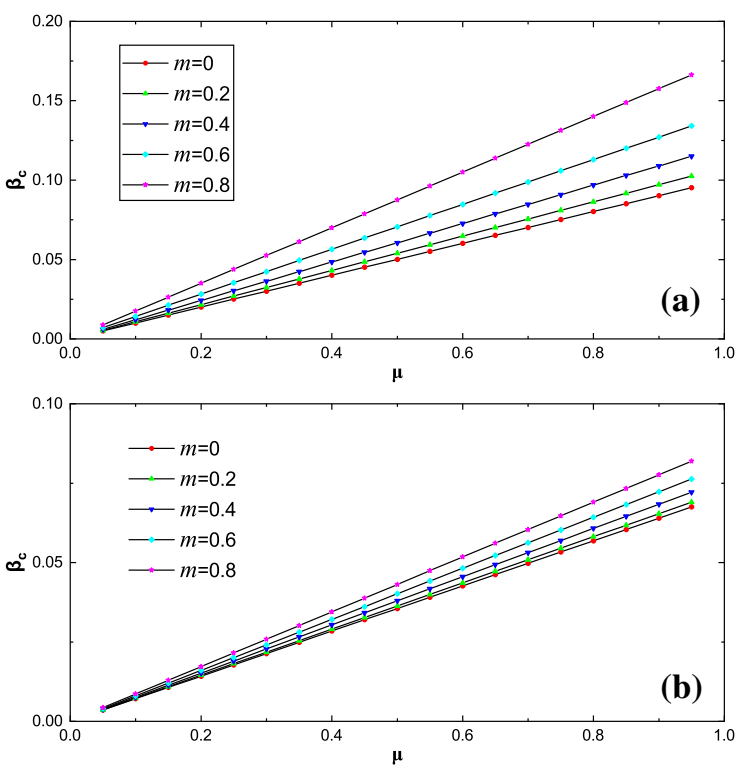

Fig. 7 Effect of $m$ and $\mu$ on the epidemic critical point $\beta_{c}$. The epidemic critical point can be raised by enhanced the propaganda intensity of the mass media and recovery rate. In panel $\mathbf{a}, \Gamma_{P}$ and $\Gamma_{N}$ is set to 0 and 0.5 . In panel $\mathbf{b}, \Gamma_{P}$ and $\Gamma_{N}$ is set to 0.3 and 0.8. Other parameters in each panel are $\lambda_{P}=\lambda_{N}=0.3$, $\delta_{P}=\delta_{N}=0.5, \xi=0.2, \kappa=0.2, \eta=0.3$ and $\omega=1$

ence the epidemic critical point through the information dissemination layer. Additionally, as illustrated in Fig. 7, $\beta_{c}$ is in direct proportion to $\mu$ for each value of $m$. This proportional relationship between $\beta_{c}$ and $\mu$ is owing to the fact that the value of $\Lambda_{\max }(M)$ is fixed when the parameters other than $\mu$ are unchanged in Fig. 7. The change of $\beta_{c}$ with $\mu$ in Fig. 7 also illustrates that easily cured epidemics are less likely to have large-scale outbreaks. Furthermore, $\beta_{c}$ in panel (b) is lower than that in panel (a) under the same conditions of $m$ and $\mu$, which shows that the epidemic that can be effectively prevented is more easily controlled.

\section{Conclusion}

In summary, through abstracting the interaction between the epidemic spreading and its related information dissemination in the real world, we devise a co-evolution model of epidemic considering two types of information under the influence of mass media based on two-layered networks. These two types of information related to epidemic prevention are positive and nega- 
tive information. The positive information guides individuals to take highly effective self-protective actions against the epidemic, while the view of negative information believes that individuals take the self-protective actions with poor effect is sufficient. Moreover, the influence of mass media on the dissemination of positive and negative information is also introduced into the proposed co-evolution model. Meanwhile, the epidemic spreading can promote the positive information dissemination, which allows more individuals to take highly effective self-protective actions against the epidemic.

To be specific, we firstly design a co-evolution model under the framework of two-layered networks to depict the co-evolution spreading of epidemics and two related information under the influence of mass media. Then, with the help of MMC, we construct the dynamical equations of the proposed co-evolution model and obtain the expression of the epidemic critical point through detailed derivations. The expression of the epidemic critical point indicates that the density of positive and negative information in the information dissemination layer at the steady state have a direct impact on the epidemic critical point. Nevertheless, the mass media can indirectly affect the epidemic critical point through its impact on information dissemination. Finally, we employ a multitude of experimental results gained by MMC calculations and MC simulations to examine the accuracy of the dynamical equations as well as expression of epidemic critical point, and then discuss the impact of mass media and two types of epidemic-relevant information on epidemic spreading. The experimental results demonstrate that accelerating positive information dissemination can greatly alleviate the epidemic size. When positive information hardly spreads, the way to accelerate the dissemination of negative information can also alleviate the epidemic to a certain extent. However, the dissemination of negative information gradually become an obstacle to epidemic control along with the dissemination of positive information becomes easier. Additionally, enhancing the propaganda intensity of the mass media and highlighting the need for taking highly effective selfprotective actions in their epidemic-relevant reports are also the effective means to prevent and control the epidemic.

The current study can offer a deeper understanding of the dynamical characteristics of the co-evolution of multiple information and epidemics under the influence of mass media. Furthermore, the results of this study also provide some useful reference for epidemic prevention and control on the basis of epidemic-relevant information dissemination, which can help individuals formulate appropriate measures to comprehend the roles of information dissemination and mass media in epidemic prevention and control under the realistic scenarios.

Acknowledgements We will acknowledge the funding support of the National Natural Science Foundation of China under Grant 61773286 and the Tianjin Research Innovation Project for Postgraduate Students under Grant 2019YJSB006.

\section{Compliance with ethical standards}

Conflict of interest The authors declare that they have no conflict of interest.

\section{References}

1. Wu, Q., Fu, X., Small, M., Xu, X.-J.: The impact of awareness on epidemic spreading in networks. Chaos 22, 013101 (2012)

2. Chinazzi, M., Davis, J.T., Ajelli, M., Gioannini, C., Litvinova, M., Merler, S., Piontti, A.P., Mu, K., Rossi, L., Sun, K., Viboud, C., Xiong, X., Yu, H., Halloran, E., Longini, I., Vespignani, A.: The effect of travel restrictions on the spread of the 2019 novel coronavirus (COVID-19) outbreak. Science 368(6489), 395-400 (2020)

3. Zhang, J., Litvinova, M., Liang, Y., Wang, Y., Wang, W., Zhao, S., Wu, Q., Merler, S., Viboud, C., Vespignani, A., Ajelli, M., Yu, H.: Changes in contact patterns shape the dynamics of the COVID-19 outbreak in China. Science 368(6498), 1481-1486 (2020)

4. Machado, J.A.T., Lopes, A.: Rare and extreme events: the case of COVID-19 pandemic. Nonlinear Dyn. 100, 2953 2972 (2020)

5. Sun, G., Wang, S., Li, M., Li, L., Zhang, J., Zhang, W., Jin, Z., Feng, G.-L.: Transmission dynamics of COVID19 in Wuhan, China: effects of lockdown and medical resources. Nonlinear Dyn. (2020). https://doi.org/10.1007/ s11071-020-05770-9

6. Perc, M., Gorišek Miksić, N., Slavinec, M., Stožer, A.: Forecasting COVID-19. Front. Phys. 8, 127 (2020)

7. Feng, S., Shen, C., Xia, N., Song, W., Fan, M., Cowling, B.J.: Rational use of face masks in the COVID-19 pandemic. Lancet Resp. Med. 8(5), 434-436 (2020)

8. Zhang, J., Dong, L., Zhang, Y., Chen, X., Yao, G., Han, Z.: Investigating time, strength, and duration of measures in controlling the spread of COVID-19 using a networked meta-population model. Nonlinear Dyn. (2020). https://doi. org/10.1007/s11071-020-05769-2

9. Li, W., Zhou, J., Lu, J.: The effect of behavior of wearing masks on epidemic dynamics. Nonlinear Dyn. (2020). https://doi.org/10.1007/s11071-020-05759-4 
10. Wang, Z., Andrews, M.A., Wu, Z.-X., Wang, L., Bauch, C.T.: Coupled disease-behavior dynamics on complex networks: a review. Phys. Life Rev. 15, 1-29 (2015)

11. Zhang, H.-F., Xie, J.-R., Tang, M., Lai, Y.-C.: Suppression of epidemic spreading in complex networks by local information based behavioral responses. Chaos 24, 043106 (2014)

12. Wang, W., Liu, Q.-H., Cai, S.-M., Tang, M., Braunstein, L.A., Stanley, H.E.: Suppressing disease spreading by using information diffusion on multiplex networks. Sci. Rep. 6, 29259 (2016)

13. Zhan, X.-X., Liu, C., Zhou, G., Zhang, Z.-K., Sun, G.-Q., Zhu, J.J.H., Jin, Z.: Coupling dynamics of epidemic spreading and information diffusion on complex networks. Appl. Math. Comput. 332, 437-448 (2018)

14. Wang, W., Liu, Q., Liang, J., Hu, Y., Zhou, T.: Coevolution spreading in complex networks. Phy. Rep. 820, 1-51 (2019)

15. Watts, D.J., Strogatz, S.H.: Collective dynamics of small world networks. Nature 393, 440-442 (1998)

16. Barabási, A.-L., Albert, R.: Emergence of scaling in random networks. Science 286(5439), 509-512 (1999)

17. Albert, R., Barabási, A.-L.: Statistical mechanics of complex networks. Rev. Mod. Phys. 74, 47-97 (2002)

18. Boccaletti, S., Latora, V., Morenod, Y., Chavez, M., Hwang, D.-U.: Complex networks: structure and dynamics. Phys. Rep. 424, 175-308 (2006)

19. Dorogovtsev, S.N., Goltsev, A.V., Mendes, J.F.F.: Critical phenomena in complex networks. Rev. Mod. Phys. 80, 1275 (2008)

20. Castellano, C., Fortunato, S., Loreto, V.: Statistical physics of social dynamics. Rev. Mod. Phys. 81, 591 (2009)

21. Wang, Z., Bauch, C.T., Bhattacharyya, S., d'Onofrio, A., Manfredi, P., Perc, M., Perra, N., Salathé, M., Zhao, D.: Statistical physics of vaccination. Phys. Rep. 664, 1-113 (2016)

22. Zhang, Z., Xia, C., Chen, S., Yang, T., Chen, Z.: Reachability analysis of networked finite state machine with communication losses: a switched perspective. IEEE J. Sel. Areas Commun. 38(5), 845-853 (2020)

23. Li, N., Wu, X., Feng, J., Xu, Y., Lü, J.: Fixed-time synchronization of coupled neural networks with discontinuous activation and mismatched parameters. IEEE Trans. Neur. Netw. Learn. Syst. (2020). https://doi.org/10.1109/TNNLS. 2020.3005945

24. Pastor-Satorras, R., Castellano, C., Mieghem, P.V., Vespignani, A.: Epidemic processes in complex networks. Rev. Mod. Phys. 87(3), 925-979 (2015)

25. Zhang, Z.-K., Liu, C., Zhan, X.-X., Lu, X., Zhang, C.-X., Zhang, Y.-C.: Dynamics of information diffusion and its applications on complex networks. Phys. Rep. 651, 1-34 (2016)

26. Yin, Q., Wang, Z., Xia, C., Dehmer, M., Emmert-Streib, F., Jin, Z.: A novel epidemic model considering demographics and intercity commuting on complex dynamical networks. Appl. Math. Comput. 386, 125517 (2020)

27. Pastor-Satorras, R., Vespignani, A.: Epidemic spreading in scale-free networks. Phys. Rev. Lett. 86, 3200-3203 (2001)

28. Liu, Z., Hu, B.: Epidemic spreading in community networks. Europhys. Lett. 72, 315-321 (2005)

29. Gross, T., D’Lima, C.J.D., Blasius, B.: Epidemic dynamics on an adaptive network. Phys. Rev. Lett. 96(20), 208701 (2006)
30. Lü, L., Chen, D.-B., Zhou, T.: The small world yields the most effective information spreading. New J. Phys. 13(12), 123005 (2011)

31. Liu, C., Zhang, Z.-K.: Information spreading on dynamic social networks. Commun. Nonlinear Sci. 19, 896-904 (2014)

32. Guo, Q., Jiang, X., Lei, Y., Li, M., Ma, Y., Zheng, Z.: Twostage effects of awareness cascade on epidemic spreading in multiplex networks. Phys. Rev. E 91(1), 012822 (2015)

33. Zheng, C., Xia, C., Guo, Q., Dehmer, M.: Interplay between SIR-based disease spreading and awareness diffusion on multiplex networks. J. Parallel Distrib. Comput. 115, 2028 (2018)

34. Hu, P., Ding, L., An, X.: Epidemic spreading with awareness diffusion on activity-driven networks. Phys. Rev. E 98(6), 062322 (2018)

35. Zhou, Y., Zhou, J., Chen, G., Stanle, H.E.: Effective degree theory for awareness and epidemic spreading on multiplex networks. New J. Phys. 21(3), 035002 (2019)

36. Wang, Z., Guo, Q., Sun, S., Xia, C.: The impact of awareness diffusion on SIR-like epidemics in multiplex networks. Appl. Math. Comput. 349, 134-147 (2019)

37. Yang, H., Gu, C., Tang, M., Cai, S.-M., Lai, Y.-C.: Suppression of epidemic spreading in time-varying multiplex networks. Appl. Math. Model. 75, 806-818 (2019)

38. Helbing, D., Brockmann, D., Chadefaux, T., Donnay, K., Blanke, U., Woolley-Meza, O., Moussaid, M., Johansson, A., Krause, J., Schutte, S., Perc, M.: Saving human lives: what complexity science and information systems can contribute. J. Stat. Phys. 158(3), 735-781 (2015)

39. Funk, S., Gilada, E., Watkinsb, C., Jansen, V.A.A.: The spread of awareness and its impact on epidemic outbreaks. Proc. Natl. Acad. Sci. USA 106, 6872 (2009)

40. Kiss, I.Z., Cassell, J., Recker, M., Simon, P.L.: The impact of information transmission on epidemic outbreaks. Math. Biosci. 225(1), 1-10 (2010)

41. Granell, C., Gómez, S., Arenas, A.: Dynamical interplay between awareness and epidemic spreading in multiplex networks. Phys. Rev. Lett. 111, 128701 (2013)

42. Boccaletti, S., Bianconi, G., Criado, R., del Genio, C.I., Gómez-Garden̄es, J., Romance, M., Sendin̄a-Nadal, I., Wang, Z., Zanin, M.: The structure and dynamics of multilayer networks. Phys. Rep. 544, 1-122 (2014)

43. de Arruda, G.F., Rodrigues, F.A., Moreno, Y.: Fundamentals of spreading processes in single and multilayer complex networks. Phys. Rep. 756, 1-59 (2018)

44. De Domenico, M., Granell, C., Porter, M.A., Arenas, A.: The physics of spreading processes in multilayer networks. Nat. Phy. 12, 901-906 (2016)

45. Wei, X., Wu, X., Chen, S., Lu, J.-A., Chen, G.: Cooperative epidemic spreading on a two-layered interconnected network. SIAM J. Appl. Dyn. Syst. 17(2), 1503-1520 (2018)

46. Zhu, P., Wang, X., Li, S., Guo, Y., Wang, Z.: Investigation of epidemic spreading process on multiplex networks by incorporating fatal properties. Appl. Math. Comput. 359, 512-524 (2019)

47. Shao, Q., Xia, C., Wang, L., Li, H.: A new propagation model coupling the offline and online social networks. Nonlinear Dyn. 98, 2171-2183 (2019)

48. Wang, Z., Xia, C., Chen, Z., Chen, G.: Epidemic propagation with positive and negative preventive information in multi- 
plex networks. IEEE Trans. Cybern. (2020). https://doi.org/ 10.1109/TCYB.2019.2960605

49. Granell, C., Gómez, S., Arenas, A.: Competing spreading processes on multiplex networks: awareness and epidemics. Phys. Rev. E 90, 012808 (2014)

50. Xia, C., Wang, Z., Zheng, C., Guo, Q., Shi, Y., Dehmer, M., Chen, Z.: A new coupled disease-awareness spreading model with mass media on multiplex networks. Inf. Sci. 471, 185-200 (2019)

Publisher's Note Springer Nature remains neutral with regard to jurisdictional claims in published maps and institutional affiliations. 